\title{
MODELADO 3D DE PRECISIÓN EN PROCESOS DE DIGITALIZACIÓN DE ESCULTURA CONSTRUIDA
}

\author{
Manuel Drago Díaz Alemán \\ Universidad de La Laguna. Dpto. Bellas Artes
}

\section{Resumen}

En la escultura contemporánea existen muchas obras que son difícilmente digitalizables en tres dimensiones utilizando procedimientos tanto activos como pasivos. Estos procedimientos plantean dificultades cuando se aplican sobre escultura construida, realizada a partir de fragmentos, con materiales laminares de poco espesor u objetos con muchas oquedades y solapamientos. Ante la imposibilidad de obtener resultados óptimos con este tipo de obras, se hace necesario utilizar nuevas estrategias que permitan la obtención de un modelo digital. Para ello se propone, ejemplificado en un objeto de estudio, la implementación de técnicas de modelado 3D de precisión. Apoyándonos en la nube de puntos obtenidas mediante sistemas activos o pasivos de digitalización 3D, se genera un modelo virtual utilizando técnicas de modelado con superficies nurbs. El resultado es un modelo 3D útil para procesos digitales de despiece, reconstrucción, así como para la catalogación o la divulgación de la obra. Abriendo la posibilidad del análisis virtual en procesos de conservación y restauración.

\section{Palabras clave: DIGITALIZACIÓN 3D; ESCULTURA CONTEMPORÁNEA; FOTOGRAMETRÍA; PROCEDIMIENTOS CONSTRUCTIVOS; MODELADO 3D}

\section{PRECISION 3D MODELLING IN DIGITISATION PROCESSES OF BUILT SCULPTURE}

\begin{abstract}
In contemporary sculpture there are many works that are difficult to digitize in three dimensions using both active and passive procedures. These procedures pose difficulties when applied to constructed sculpture, made from fragments, with thin sheet materials or objects with many cavities and overlaps. Given the impossibility of obtaining optimal results with this type of work, it is necessary to use new strategies that allow obtaining a digital model. For this, it is proposed, exemplified in an object of study, the implementation of precision 3D modeling techniques. Based on the point cloud obtained through active or passive 3D digitization systems, a virtual model is generated using modeling techniques with nurbs surfaces. The result is a 3D model useful for digital processes of cutting, reconstruction, as well as for cataloging or disseminating the work. Opening the possibility of virtual analysis in conservation and restoration processes.
\end{abstract}

Keywords: 3D DIGITIZATION; CONTEMPORARY SCULPTURE; PHOTOGRAMMETRY; CONSTRUCTION PROCEDURES; 3D MODELING

\footnotetext{
Díaz Alemán. Manuel Drago. 2021. "Modelado 3D de precisión en procesos de digitalización de escultura construida". AusArt 9 (2): 113-125. DOI: 10.1387 /ausart.23077
}

AUSART 


\section{INTRODUCCIÓN}

Desde el siglo XIX, la fotografía ha sido una herramienta básica y eficaz para su uso en Conservación y Restauración (CyR) de obras de patrimonio cultural. Sigue siendo de uso extendido, y se muestra imprescindible para documentar objetos bidimensionales como pinturas, papel y tejidos. Pero cuando un objeto tiene una mayor complejidad geométrica, como es el caso de la escultura, la fotografía suele ser insuficiente y en muchos casos inadecuada para la tarea de registro y documentación.

El carácter tridimensional de un objeto requiere, a diferencia de uno bidimensional, de un sistema de medición que pueda abordar, de manera global, la multiplicidad de medidas necesarias para el registro de sus tres dimensiones, por complejo que este sea. Este sistema de medición sólo es posible mediante la implementación de herramientas digitales, lo que conocemos como digitalización 3D que, a diferencia de la 2D, basada en una imagen raster (matriz de pixeles), permite la obtención de datos de profundidad, es decir, mediciones tridimensionales. Estos datos deben ser lo más precisos posibles ya que la falta de precisión puede conducir a una pérdida de información y como resultado, a una interpretación errónea de la realidad de la pieza (Guidi \& Frischer 2020).

Con un sistema 3D, a la posibilidad del registro con precisión de todas las medidas de un objeto, se le añade la posibilidad de registrar, de manera simultánea la información de color $(R G B)$ de su superficie, de tal manera que es posible la construcción de un modelo virtual que incluya los acabados y policromía, ubicándolos de manera coincidente con su geometría.

Además de la precisión, debemos destacar la maleabilidad como una de las principales características del modelo digital tridimensional. El examen de un objeto patrimonial, a través de su modelo digital 3D permite medir, seccionar, descomponer, recomponer, comparar e incluso realizar análisis estructurales del objeto, de una forma que no sería posible con el objeto físico real. Cada uno de estos usos implica una virtualización, es decir la creación de un modelo alternativo virtual y tridimensional que permite obviar la manipulación del objeto real, salvaguardando su integridad en todo momento. Este modelo virtual puede ser de utilidad para la divulgación mediante la construcción de réplicas virtuales o físicas del objeto de patrimonio (Saorín et al. 2017). 


\section{LA APARICIÓN DE LA ESCULTURA CONSTRUIDA}

Con la entrada en el siglo XX, asistimos a lo que la mayoría de los autores consideran la aparición de la escultura contemporánea, bajo la influencia de movimientos artísticos como el Cubismo, el Futurismo y el Constructivismo, abandonando las convenciones heredadas que definían la escultura desde hacía siglos. Desde sus orígenes, la escultura había asumido como tema principal, la representación de la figura humana, que se materializaba como un cuerpo sólido y opaco. La escultura moderna comenzó por negar esta finalidad, prescindiendo de la solidez, y sirviéndose de planos y líneas que empezaban a definir vacíos (Maderuelo 2012, 18). La noción de espacio adquirió importancia frente a la idea tradicional de volumen. Despojándose de su función monumental, dio el primer paso de su paulatino proceso de expansión (Krauss 1979). Al mismo tiempo, abandonó convencionalismos como la obligada inspiración en la naturaleza, el estilo realista, los materiales nobles y duraderos, y las técnicas tradicionales de la talla y modelado. El abandono de la figuración problematiza la dicotomía espacio volumen, entendiendo que el espacio no es ya sólo el volumen encerrado por la superficie de la obra, sino que se hace extensivo a su alrededor.

Desde principios del siglo XX y a lo largo de este, los escultores se apropian, además, de una nueva materialidad y por consiguiente de nuevos procedimientos provenientes de la industria. La evolución de las formas es decisiva cuando empiezan a ser obtenidas por la utilización de nuevas técnicas industriales como el cizallado, el plegado, el atornillado, el remachado y la soldadura. "Estos nuevos materiales y sus técnicas específicas, que nos alejan de los tradicionales procedimientos de quitar, propios de la talla, y de añadir, en que se basa el modelado, nos sugieren otro verbo: <construir>, lo cual supone pensar, proyectar y prever de antemano materiales heterogéneos que hay que medir, cortar y ensamblar con precisión" (Maderuelo 2012, 26).

\section{EL RETO PARA EL RESTAURADOR}

Es esa escultura <construida> uno de los grandes hallazgos de la Vanguardias Históricas, permitiendo al escultor una mayor versatilidad en el uso de diferentes materiales, una reducción en el tiempo de ejecución de las obras 
y el aumento del tamaño de las mismas. Este cambio que atañe a todos los aspectos del hecho escultórico, debe ser asumido también por el restaurador, a la hora de plantear sus estrategias de intervención, incluidas las de digitalización. Los avances tecnológicos abren nuevas posibilidades que deben estudiarse, asumiendo que "es un reto para el sentido de la responsabilidad del restaurador decidir cuánto de lo que es posible hacer a través de la ciencia y la tecnología debe utilizarse" (Althöfer 1981).

El arte contemporáneo y por ende la escultura, reclama especialización y conocimiento por parte del restaurador, al que le plantea continuos retos que afectan a las directrices metodológicas y principios de actuación de la propia teoría de la CyR, definida desde 1964 (Santabárbara 2016, 141). La CyR como disciplina, se debate en torno a los enfoques y a los medios con los que abordar muchas de estas obras.

\section{DIGITALIZACIÓN 3D}

Es cada vez más extendido el uso de las tecnologías de digitalización tridimensional, que procedentes de ámbitos técnicos, como la Ingeniería o la Topografía, han sido adoptadas por ciencias como la Arqueología, la Paleontología, la Historia del Arte y la CyR de bienes culturales (Guidi \& Frischer 2020). Esta apropiación multidisciplinar de las tecnologías de digitalización 3D, hace necesaria la aclaración de algunos términos ya que pueden prestarse a confusión debido al uso diferente que se hace de ellos en gran parte de la literatura especializada.

Entenderemos por digitalización a los procedimientos que, haciendo uso de tecnologías digitales, sirven para la realización de una réplica digital de un objeto real. Esta réplica puede ser de utilidad en CyR para la catalogación, la difusión, la reconstrucción y restauración virtual o incluso el análisis mediante el uso de software especializado (Fuentes Porto, y otros 2021). Si esta réplica es capaz de registrar información tridimensional del objeto de referencia, hablaremos de digitalización 3D, quedando su clasificación de la siguiente manera. (Fig.1)

Las técnicas de contacto como CMM, han sido paulatinamente sustituidas por las que no precisan de contacto con la obra ya que estas ofrecen numero- 
sas ventajas, como la posibilidad de trabajar sin contacto físico con el objeto, evitando posibles daños o alteraciones y la mayor velocidad en la captura de datos. Estas, a su vez, se dividen en activas o pasivas. Los sistemas activos se caracterizan por la utilización de dispositivos de captura que, si bien han disminuido su coste en los últimos años, siguen siendo, menos accesibles que los pasivos, que utilizan un dispositivo fotográfico. En nuestro objeto de estudio hemos utilizado, uno de estos sistemas pasivos, en concreto la fotogrametría.

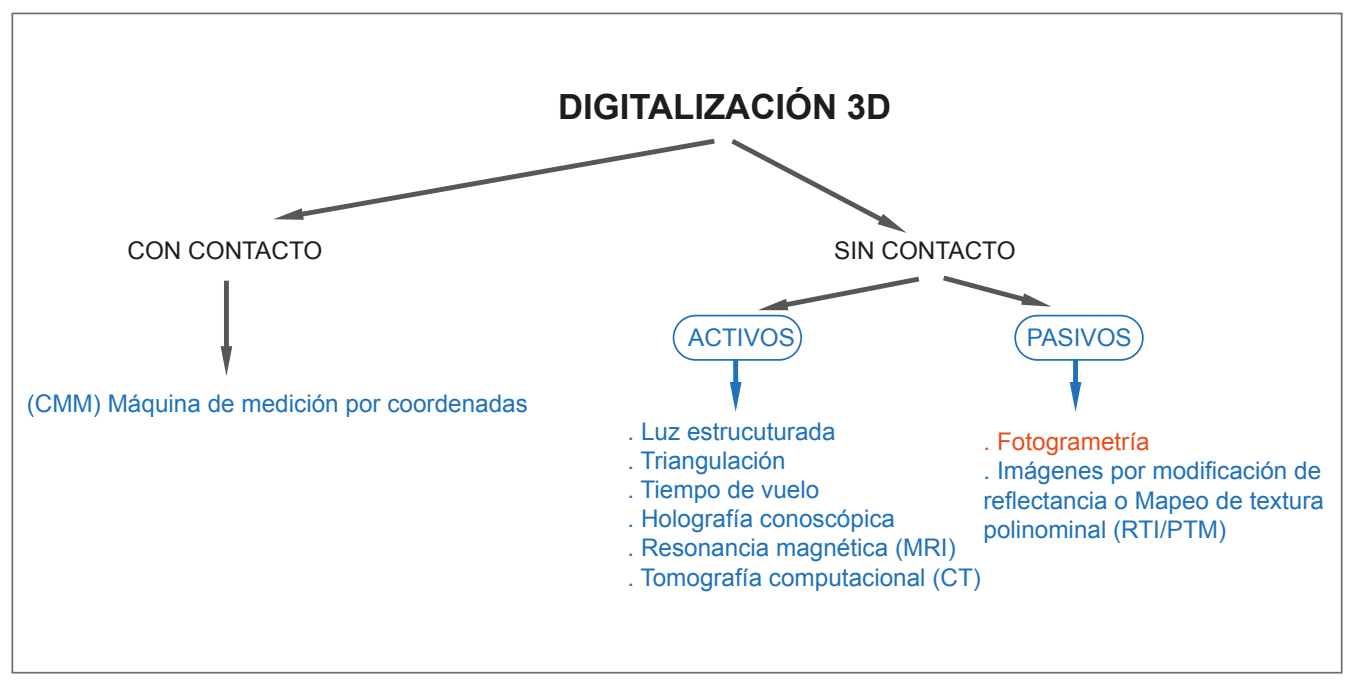

Fig.1 Esquema de clasificación de las tecnologías de digitalización 3D. En naranja, una de las técnicas comparadas en este trabajo.

La fotogrametría es, debido al bajo coste de los dispositivos fotográficos y a la estandarización del software fotogramétrico, la técnica más utilizada para la digitalización tridimensional de objetos (Morita y Bilmes 2018, 4). Es una técnica que se utiliza para determinar la geometría tridimensional, es decir, ubicación, tamaño y forma de los objetos físicos mediante la medición y el análisis de sus imágenes bidimensionales. Cuando el tamaño del objeto y la distancia entre este y la cámara son ambos inferiores a $100 \mathrm{~m}$, se define como fotogrametría de corto alcance. Este es el tipo de fotogrametría adecuado para su uso en CyR de bienes culturales (Jiang, Jáuregui \& White 2008, 824). Los datos que obtenemos, provienen de las zonas comunes en las diferentes imágenes utilizadas, zonas de solape o recubrimiento que propician una visión estereoscópica. Con la utilización de un software especializado, podemos obtener, a partir de estas coincidencias, una nube de puntos que registra la información tridimensional y que, posteriormente, servirá de base para la construcción de 
un modelo tridimensional de malla poligonal. El modelo virtual obtenido con esta técnica tiene unas proporciones y apariencia, acordes con la realidad.

La digitalización mediante escáner o fotogrametría obtiene resultados óptimos cuando se aplican sobre objetos de bulto redondo, formas orgánicas, muchas veces figurativas cuyos acabados son mayoritariamente opacos y poco reflectantes. En esta casuística entran gran parte de la obra escultórica de los fondos culturales, desde restos arqueológicos hasta la estatuaria neoclásica. Sin embargo, las técnicas de digitalización 3D, tanto pasivas como activas, plantean dificultades cuando se aplican sobre objetos realizados a partir de fragmentos, con materiales laminares de poco espesor u objetos con muchas oquedades. Los acabados también pueden ser problemáticos, superficies monocromas muy lisas, superficies oscuras muy poco reflectantes o, por el contrario, superficies muy reflectantes o transparentes (Guidi, Gonizzi \& Micoli 2015). A causa de estas dificultades, se producen grandes aberraciones en la construcción de la nube de puntos, lo que imposibilita la construcción de una maya poligonal que responda a la forma deseada.



(Fig. 2) Esquema que propone una nueva clasificación de las técnicas de digitalización. En naranja las técnicas comparadas en este trabajo.

Ante la imposibilidad de obtener resultados óptimos con este tipo de obras, se hace necesario abordar nuevas estrategias que permitan la obtención de un modelo digital. Es entonces cuando parece plausible la implementación 
de las técnicas existentes de digitalización 3D mediante la incorporación del modelado 3D de precisión. Este término alude a aquellas técnicas digitales de modelado 3D geométrico, de uso generalizado en disciplinas como Ingeniería y Arquitectura. Estas técnicas trabajan con superficies nurbs o sólidos geométricos, que son entidades matemáticas alternativas a la malla poligonal. De esta manera la clasificación de estas tecnologías respondería a una nueva taxonomía. En relación a su funcionamiento, estas técnicas podríamos clasificarlas, por un lado, como de captura de datos que aglutinarían a las que se realizan con y sin contacto y, por otro lado, como de replicado mediante modelado 3D. Estas últimas son dependientes de las primeras ya que se realizarán partiendo de la nube de puntos obtenidas mediante captura de datos (Fig. 2).

\section{OBJETO DE ESTUDIO}

Para ilustrar la viabilidad de este proceso se ha acometido la digitalización y posterior reconstrucción digital de la obra del escultor colombiano Edgar Negret (1920-2012) titulada Sol Rojo (Fig. 3). Está ubicada en la ciudad de Santa Cruz de Tenerife y fue construida con motivo de la II Exposición Internacional de Escultura en la Calle. Se trata de una obra emblemática de este autor y refleja muchas de las características que a nivel procedimental definen gran parte de la producción del autor, que se caracteriza por el uso de elementos geométricos mediante la construcción de obras en hierro y aluminio, uniendo sus láminas mediante tuercas y tornillos (Salvador 1992).

\begin{tabular}{|l|l|}
\hline AUTOR & Edgar Negret \\
\hline TÍTULO & Sol Rojo \\
\hline AÑO & 1995 \\
\hline DIMENSIONES & $399 \times 103 \times 391,5 \mathrm{~cm}$. \\
\hline MATERIAL & $\begin{array}{l}\text { Pancha de aluminio de } 5 \mathrm{~mm} . \\
\text { de espesor }\end{array}$ \\
\hline TÉCNICA & \begin{tabular}{l} 
Plegado, atornillado y lacado \\
\hline UBICACIÓN
\end{tabular} \\
\hline
\end{tabular}

Fig. 3 Datos más relevantes de la obra escogida. 
Se ha optado por esta obra como objeto de estudio ya que, por su forma (múltiples recovecos y solapamientos) y por su procedimiento constructivo, (plancha de aluminio plegada y atornillada), plantea serias dificultades para ser digitalizada tanto mediante técnicas activas como pasivas.

En este trabajo, hemos optado por usar programas informáticos especializados y muy conocidos, pero de costo accesible. Para el procesado fotogramétrico se escogió el programa Agisoft Metashape, anteriormente conocido como Photoscan, en su versión más básica destinada a la fotogrametría de objeto cercano. Es un programa muy habitual en los centros superiores de educación de CyR de Bienes Culturales y Arqueología, con una licencia para usos académicos, de muy bajo coste. Para la realización del modelado de precisión hemos optado por el programa Rhinoceros 7, un software de uso muy estandarizado en espacios educativos y laboratorios de Ingeniería, Diseño y Fabricación Digital, cuya licencia educativa es muy asequible.

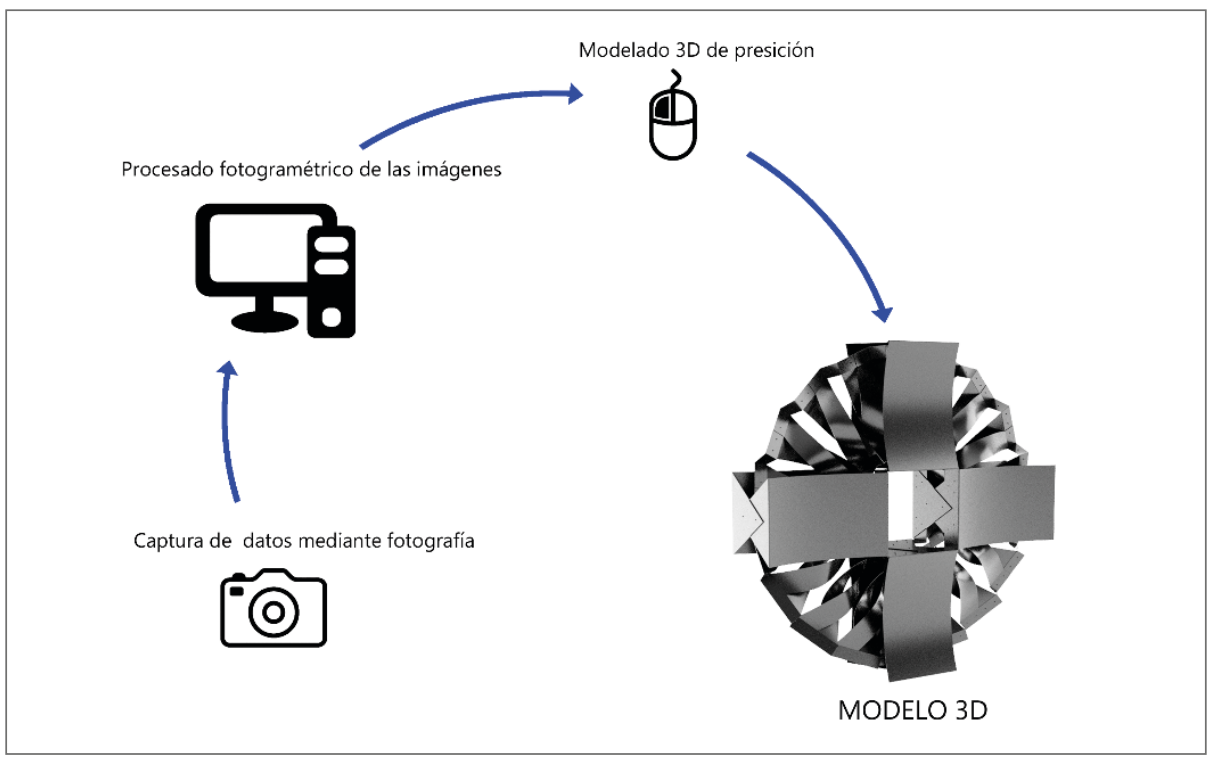

Fig.4 Esquema del proceso de trabajo

El proceso de trabajo viene marcado por tres etapas consecutivas, la adquisición de datos, el procesado fotogramétrico y el modelado 3D de precisión (Fig. 4). Esta última etapa es la más significativa del proceso. El software elegido es un modelador nurbs, un modelo de representación matemática óptimo en los procesos de ingeniería inversa, que nos permite construir un modelo digital a partir de uno físico existente al que se le han tomado mediciones reales 
con la finalidad de saber de qué elementos se compone y cuál fue su proceso fabricación (Yin 2004).

La adquisición de datos se realizó mediante captura fotográfica utilizando una cámara Canon EOS $700 D$ con una distancia de foco fija de $35 \mathrm{~mm}$. Se realizaron un total de 75 imágenes en formato $R A W$ y resolución de $10 \mathrm{Mpx}$., que se dispusieron de manera circular alrededor de la obra. Al tratarse de una escultura ubicada al aire libre, se optó por realizar las imágenes un día nublado, evitando de esta manera la luz intensa y la presencia de sombras arrojadas sobre la pieza.

Para el procesado fotogramétrico se utilizó una CPU Intel(R) Core(TM) i7-6700K 4.00G Gz con 32 Gb de memoria RAM y una memoria interna sólida de $1 T$ b. con sistema operativo Windows 10.

El software utilizado fue Agisoft Metashape, capaz de realizar todo el flujo de trabajo, empezando por la orientación de las imágenes, continuando con la creación de la nube de puntos, la construcción de la maya poligonal y terminando por la construcción de la textura y su mapeado. Todo este proceso fue llevado a cabo con los valores que por defecto incorpora el programa y ajustado el nivel de resolución alto. El resultado fue una nube de puntos densa de 1.854.756 puntos, que fue escalada mediante puntos de control y exportada en formato $P L Y$, incluyendo la información de color asignada a cada punto. Esta nube de puntos es incapaz de registrar toda la información geométrica de forma correcta ya que el objeto es laminar con sólo $5 \mathrm{~mm}$ de espesor y formalmente complejo, con la inclusión de muchos solapamientos (Fig. 5).

Para el modelado 3D de precisión se utilizó el mismo equipo informático que en la fotogrametría. El software utilizado fue Rhinoceros 7 de (Robert McNeel \& Associates), un modelador de superficies nurbs de alta precisión. El proceso de modelado hace uso de la nube de puntos como referencia espacial sobre la que acometer el modelado de las distintas piezas. Este proceso se ayuda también de algunas mediciones directas tomadas sobre la obra original (Fig.6).

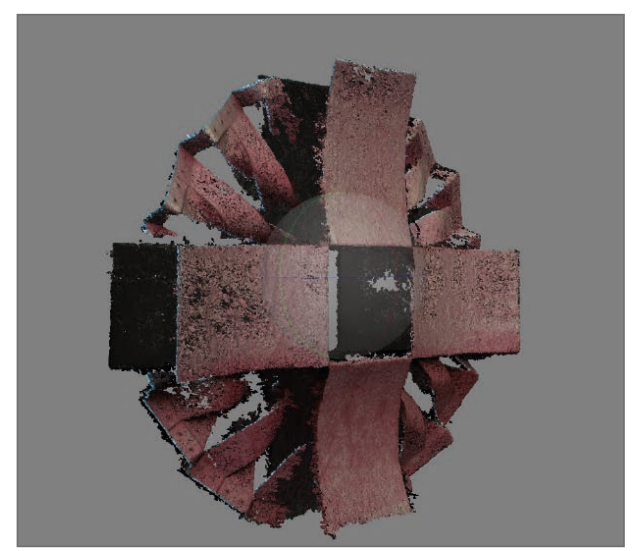

Fig. 5 Imagen de la nube de puntos obtenida. 


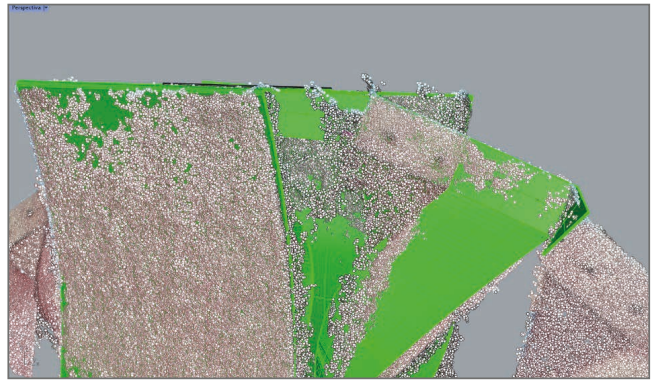

Fig. 6 Detalle del proceso de modelado a partir de la nube de puntos. En verde el modelo generado por superficies nurbs.
El modelo se realizó con una precisión de centésimas de milímetro. Para resolver el modelado se utilizaron múltiples herramientas, predominando las de generación y extrusión de superficies y la matriz polar. La elección de estas herramientas viene determinada por la tipología de la pieza, en este caso, construcción laminar dispuestas simétricamente de forma radial.

\section{RESULTADOS Y DISCUSIÓN}

En el caso aquí expuesto, que responde a una de las tipologías frecuente de la escultura construida, se puede comprobar como las mallas poligonales generadas por fotogrametría plantea numerosos errores y no es capaz de describir la geometría de la escultura real. El delgado espesor de las planchas de aluminio $(5 \mathrm{~mm})$ y la propia forma de la escultura, que define multitud de huecos internos, hace que el programa la interprete como formas cerradas. Las formas abiertas y las zonas oscuras propiciadas por los solapes, se traducen en una pérdida importante de datos (Fig.7).

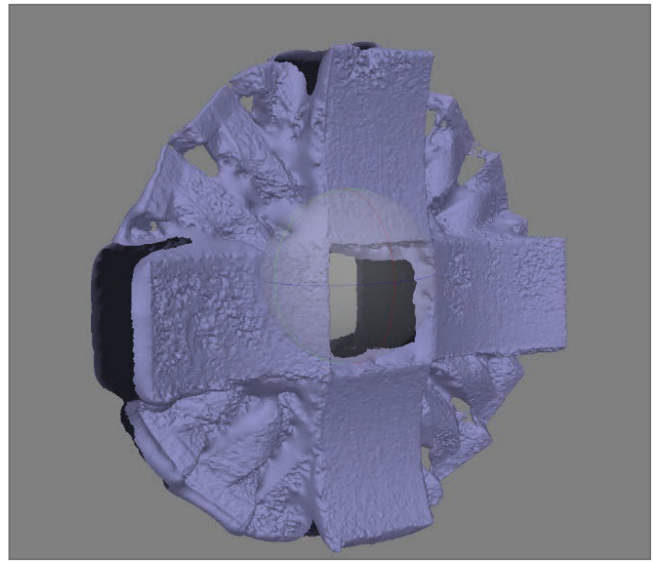

(Fig. 7) Imagen de la malla poligonal obtenida en Agisoft Metashape.
Sin embargo, esta nube de puntos si es capaz de registra la posición real relativa que defina parte de la forma de manera proporcionada. Si asignamos una medida real de referencia, podemos obtener la posición real relativa de muchos puntos que pueden servir de referencia para acometer el modelado 3D de piezas imposibles de digitalizar mediante captura de datos.

El resultado fue un modelado $3 D$ de alta precisión formado por un total de 
116 Piezas que responden a 10 tipologías distintas más la tornillería completa con 444 unidades (Fig. 8).



(Fig. 8) Imagen de despiece con el número de unidades por tipología.

De la misma manera que a lo largo del siglo $X X$ la escultura contemporánea adoptó materiales y procedimientos industriales. A lo largo del siglo XXI, los escultores continúan apropiándose de nuevos procesos estandarizados por la industria como los sistemas de diseño y fabricación asistidos por ordenador (CAD/CAM). Si tal y como se propone en este trabajo, puede ser útil la adopción de herramientas de modelado 3D de precisión para la digitalización de obras contemporáneas del pasado siglo, la adopción de estas herramientas en las futuras restauraciones de obras actuales será necesaria, ya que las esculturas de hoy incluyen en sus procesos de diseño y fabricación los sistemas CAD/CAM. Esto implica la existencia de archivos digitales con la información tridimensional exacta en el momento de su fabricación, que deberán ser conservados para su uso en previsibles futuras acciones de conservación y restauración.

La inclusión de técnicas de modelado 3D de precisión, como técnica de documentación de bienes culturares puede justificarse desde el punto de vista de las nuevas teorías de la CyR, si atendemos a las propuestas de conservación evolutiva, que definen la recreación como una estrategia que re-produce, a la vez que documenta, el bien patrimonial, generando una versión susceptible de conservarse evolutivamente (García Morales 2019, 26) . 


\section{CONCLUSIONES}

La utilización de técnicas de modelado de precisión implica un grado de especialización mayor que las de captura de datos, ya que obliga al conocimiento de interfaces de modelado afines al campo del Diseño del producto o la Ingeniería. Si bien es posible que el restaurador aprenda su uso, sería recomendable optar por el trabajo colaborativo en equipos multidisciplinares.

Si bien, los sistemas de digitalización basados en la réplica por modelado 3D no son capaces de registrar el estado actual de conservación de una obra, son imprescindibles en la elaboración de modelos digitales de obras de formas complejas no digitalizables por medios de captura de datos. Así mismo, son imprescindibles para abordar digitalmente obras formadas por multitud de piezas menores tipo acumulación o ensamblaje, permitiendo el despiece virtual.

Los modelos obtenidos por esta técnica, podrán ser sometidos a pruebas de análisis de elementos finitos (CEF), con la utilización de software especializado. Este tipo de análisis puede partir de modelos digitales de objetos reales para predecir su reacción a fuerzas como la gravedad, el viento o vibraciones, los cambios de temperatura, flujo de líquidos y otros efectos físicos en condiciones reales. Lo que puede ser de gran utilidad en el desarrollo de estrategias de conservación preventiva, sin alteración alguna de la obra original.

Por todo ello, sería conveniente incluir las técnicas de replicado mediante modelado 3D en la taxonomía general de las técnicas de digitalización tridimensional de obras de patrimonio.

\section{Referencias bibliográficas}

Althöfer, Heinz. 1981. "Historical and ethical principles of restoration". In ICOM Committee for Conservation 7th Triennial Meeting: Ottawa, Canada 21-25 September. Paris: The International Council of Museums

Fuentes Porto, Alba, Elisa Díaz González, Manuel Drago Díaz Alemán \& Esteban Manuel Amador García. 2021. "Proceso de registro y seguimiento de daños morfológicos en metal arqueológico mediante documentación 3D". GE-Conservación 19: 309-19. https://doi. org/10.37558/gec.v19i1.1002

García Morales, Lino. 2019. Teoría de la conservación evolutiva: Conservación y restauración del arte de los nuevos medios. Norderstedt: Books on Demand 
Guidi, Gabriele \& Bernard D. Frischer. 2020. "3D digitization of cultural heritage". In 3D imaging, analysis and applications, editors, Yonghuai Liu, Nick Pears, Paul L. Rosin \& Patrik Huber. Cham, Switzerland: Springer. https://doi.org/10.1007/978-3-030-44070-1_13

Guidi, Gabriele, Sara Gonizzi Barsanti \& Laura Loredana Micoli. 2014. "Image pre-processing for optimizing automated photogrammetry performances". ISPRS Annals of the Photogrammetry, Remote Sensing and Spatial Information Sciences II(5): 145-52. https://doi. org/10.5194/isprsannals-II-5-145-2014

Jiang, Ruinian, David V. Jáuregui \& Kenneth R. White. 2008. "Close-range photogrammetry applications in bridge measurement: Literature review". Measurement 41(8): 823-34. https://doi.org/10.1016/j.measurement.2007.12.005

Krauss, Rosalind. (1979) 1985. "La escultura en el campo expandido". En La posmodernidad, Jean Baudrillard et al.; selección y prólogo de Hal Foster; traducción, Jordi Fibla, 59-74. Barcelona: Kairós

Maderuelo Raso, Javier. 2012. Caminos de la escultura contemporánea. Salamanca: Universidad de Salamanca

Morita, María Mercedes \& Gabriel Mario Bilmes. 2018. "Applications of low-cost 3D imaging techniques for the documentation of heritage objects". Óptica Pura y Aplicada 1-11. https:// doi.org/10.7149/OPA.51.2.50026

Salvador González, José María. 1991. Edgar Negret: De la máquina al mito. Monterrey: Edisigma

Santabárbara Morera, Carlota. 2016. "La conservación del arte contemporáneo ¿Un desafío para la teoría de la restauración crítica?". En Conservando el pasado, proyectando el futuro: Tendencias en la restauración monumental en el siglo XXI [Preserving the past, projecting the future: Tendences in 21st Century monumental restoration], coord. por Ascensión Hernández Martínez, 141-56. Zaragoza: Institución Fernando el Católico. https://ifc.dpz.es/recursos/publicaciones/35/83/09santabarbara.pdf

Saorin Pérez, José Luis, Carlos Carbonell Carrera, Jorge de la Torre Cantero, Cecile Meier \& Drago Diaz Alemán. 2017. "Three-dimensional interpretation of sculptural heritage with digital and tangible 3d printed replicas". TOJET 16(4): 161-9. http://www.tojet.net/articles/ v16i4/16415.pdf

Yin, Zhongwei. 2004. "Reverse engineering of a NURBS surface from digitized pointssubject to boundary conditions". Computers \& Graphics 28(2): 207-12. https://doi.org/10.1016/J. CAG. 2003.12.007 